\title{
Mechanical-sensorless induction motor drive based only on DC-link measurements
}

\author{
M.O. Sonnaillon, G. Bisheimer, C. De Angelo, J. Solsona and G.O. García
}

\begin{abstract}
Sensor count reduction for high-performance induction motor (IM) drives is considered. A novel strategy to compute the three phase currents, based on a single current sensor and an adaptive observer, is proposed. The observer estimates the motor state variables, allowing then implementing an IM field-oriented controlled (FOC) drive with closed speed loop based only on DC-link measurements. To demonstrate the practical feasibility of this proposal, representative experimental results, obtained with a FOC experimental prototype, are presented. The FOC prototype showed results similar to those from a conventional FOC drive with individual phase current sensors. The sensor count reduction achieved represents a significant cost diminution in the implementation of low-power high-performance drives.
\end{abstract}

\section{Introduction}

High-performance induction motor (IM) drives need the instantaneous values of the stator currents, the magneticflux vector position and the rotor speed value in order to work properly. These variables are usually measured and/or estimated using current, voltage and/or speed sensors, depending on the particular implementation. A reduction in the number of these sensors is desirable to reduce cost, simplify hardware and increase reliability [1]. However, sensor count reduction should be performed without either affecting the system performance or increasing excessively the hardware or software complexity.

Phase current measurements are usually performed by using Hall-effect sensors or sensing resistors (SR) with the appropriate signal conditioning circuits. In a standard threephase inverter, the phase currents can be reconstructed using the inverter switches states and a single current sensor placed in the DC-link [1-8]. In [2] a phase current reconstruction method using a single DC-link current sensor is proposed. The basic principle is described with useful hardware considerations. Furthermore, it is shown that, depending on the pulse-width-modulation (PWM) strategy, the DC-link current acquisitions are impossible at some PWM cycles because of the too short time each phase current is circulating through the DC-link. In [3] a statorflux oriented controller using a single current sensor is presented. A special modification is proposed in the space vector modulation (SVM) pattern in order to achieve the required DC-link current acquisitions. In [4] an SVM

(C) The Institution of Engineering and Technology 2006

IEE Proceedings online no. 20050552

doi:10.1049/ip-epa:20050552

Paper first received 28th July 2005 and in final revised form 23rd March 2006 M.O. Sonnaillon, G. Bisheimer, C. De Angelo and G.O. García are with the CONICET, and also with Grupo de Electrónica Aplicada (GEA), Facultad de Ingeniería, UNRC, Ruta Nacional \#36 Km. 601, X5804BYA, Río Cuarto, Argentina

J. Solsona is with CONICET and also with the Departamento de Ingeniería Eléctrica y de Computadoras, Instituto de Investigaciones en Ingeniería Eléctrica ‘Alfredo Desages', UNS, Av. Alem 1253 (8000) Bahía Blanca, Argentina

E-mail: msonnaillon@ing.unrc.edu.ar pattern modification (SVM-PM) that produces a lower current harmonic content is proposed.

The SVM-PM, required to reconstruct the phase currents, increments the power losses, the audible noise and the high-frequency content in voltages and currents $[5,6]$. Furthermore, the inverter output-voltage is reduced by the SVM-PM in the overmodulation operating mode [9].

To reduce the negative effects, methods based on the phase current estimation have been proposed. In [7] a scheme based on the stator circuit model and a least-squares recursive method is proposed to estimate the phase currents when they cannot be reconstructed. In [5] a comparison of three different single current sensor strategies applied to a three-phase controlled rectifier is presented. In [6] a predictive current controller is applied to an AC motor drive where the phase currents are estimated using three individual observers. These observers need the induced electromotive force (EMF) of the motor, which must be estimated independently.

The above-mentioned methods, based on estimators, were proposed to avoid or at least reduce the SVM-PMs. However, in some operating conditions, like overmodulation or low modulation indexes [10], the current estimation methods will not produce accurate estimations unless they are combined with a SVM-PM scheme.

Regarding the shaft speed, it is usually measured using a tachogenerator, an optical encoder or a resolver. However, these mechanical sensors can be avoided using a speed estimator, which is generally based on stator voltage and current measurements. Several speed estimation strategies can be found in the literature [11]. One of the most studied is the speed estimator proposed by Kubota et. al. [12], which computes an estimation of the rotor speed using an adaptive observer.

With regard to the IM phase voltages, required by the state variable estimators, they can be directly measured using an AC voltage sensor in each phase (such as a Halleffect or isolated amplifier). They can also be reconstructed from the DC-link voltage and the inverter switches states, using a single voltage sensor [13].

To the best knowledge of the authors, a single current sensor method capable of operating the inverter in overmodulation mode is only briefly mentioned in [8]. 
A detailed solution of a phase currents computation method capable of operating in the whole modulation range has not been published yet.

\section{Phase currents computation}

As it was mentioned in Section 1, in some cases, the phase current reconstruction is not possible using a conventional SVM pattern because of the too short available time to acquire the DC-link currents. Hence, an SVM-PM must be performed to allow the phase currents reconstructions. However, the SVM-PM has negative effects for the drive, such as an increment in the power losses and a reduction of the output RMS voltage. The importance of these effects depends on each particular application; but in any case they should be reduced to improve the drive performance.

In this Section, a strategy to compute the IM phase currents is proposed. This strategy is based on a particular SVM-PM, valid in the whole modulation range, working together with a state observer to reduce the SVM-PM negative effects. The observer operates based on the stator voltages reconstructed from a single DC-link voltage sensor. The same state observer also estimates the IM rotor flux and rotor speed that can be used to implement a highperformance drive, as will be shown in Section 3.

The proposed idea is shown in Fig. 1. In the first computing cycle, a SVM-PM is performed to allow the DClink current acquisitions and the corresponding phase current reconstruction, but during the next $(N-1)$ cycles, the phase currents are estimated using the observer.

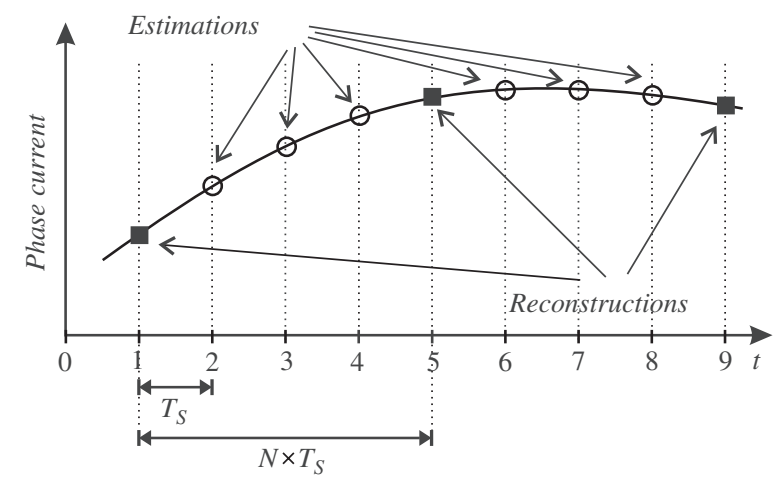

Fig. 1 Illustration of proposed phase currents computation scheme Phase currents are reconstructed one of each $N$ cycles. Between reconstructions, the phase currents are estimated

\subsection{Phase current reconstruction}

A simplified schematic diagram of a three-phase inverter is shown in Fig. 2a. The inverter state is given by the combination of the states of the switches (represented in the Figure by $S a, S b$ and $S c$ ). In every non-zero state (i.e. active current flow), the instantaneous current that flows through the DC-link is equal in magnitude to one of the three output phase currents. Based on this fact, the phase currents can be reconstructed using a single DC-link current sensor and the states of the inverter.

The SVM is one of the most widely used strategies in three-phase inverters because it can produce a minimum number of commutations per cycle or minimum current ripple, while allow high modulation indexes (including overmodulation) [10]. In conventional SVM patterns, the inverter takes two different active states at each switching cycle. Therefore, two different phase currents can be

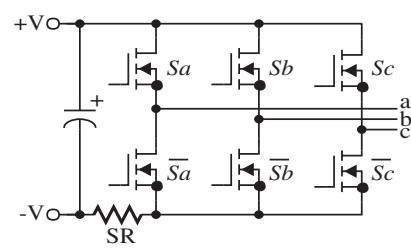

$a$

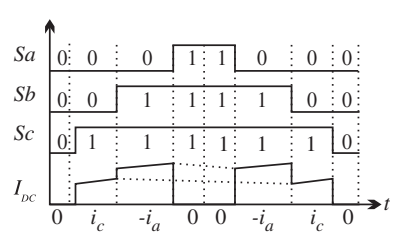

$b$
Fig. 2

$a$ Three-phase MOSFET inverter simplified schematic diagram (antiparallel diodes are omitted for simplicity)

$b$ Example of inverter switching states and corresponding DC-link current for single cycle of SVM, where two different phase currents can be acquired

acquired in each cycle using the DC-link sensor. Figure $2 b$ shows an example of a SVM cycle and the ideal DC-link current waveform. The plotted state sequence is:

$$
000 \rightarrow 001 \rightarrow 011 \rightarrow 111 \rightarrow 111 \rightarrow 011 \rightarrow 001 \rightarrow 000
$$

where 000 and 111 are the called zero states.

The third phase current can be computed knowing that the sum of the three phase currents is zero without neutral connection. The correspondence between phase currents and DC-link current, for each inverter state, is given in Table 1.

Table 1: Correspondence between phase currents and DC-link current for each inverter state

\begin{tabular}{ll}
\hline Inverter state & DC-link current \\
\hline 100 & $i_{a}$ \\
110 & $-i_{c}$ \\
010 & $i_{b}$ \\
011 & $-i_{a}$ \\
001 & $i_{c}$ \\
101 & $-i_{b}$ \\
111 and 000 & 0 \\
\hline
\end{tabular}

In some cases, the duration of each SVM state is shorter than the minimum time required to acquire the DC link current $\left(T_{M I N}\right)$, and consequently the phase currents cannot be accurately reconstructed. Some factors that affect this time are the DC-link layout, the time response of the acquisition circuits, such as analogue-to-digital (A/D) converter, the signal conditioning amplifiers and the dynamic response of the current sensor (e.g. SR), as is shown in Section 3. There are three special cases for which the available acquisition time is too short:

Case A: The voltage reference is in the same direction as one of the six fundamental vectors. In this case, the state of the inverter alternates between a zero and a non-zero state for almost the whole SVM cycle. As a second non-zero state is not active for enough time to carry out an acquisition, only one phase current can be acquired. The vector diagram of Fig. $3 a$ represents the SVM vector voltage reference associated with the available DC-link current acquisition time, where the darker grey levels correspond to lower available acquisition times for a conventional SVM routine. Case B: Low modulation index. In this case the inverter remain in zero state for most of the cycle duration, and the non-zero vectors remains active for too short a time. 


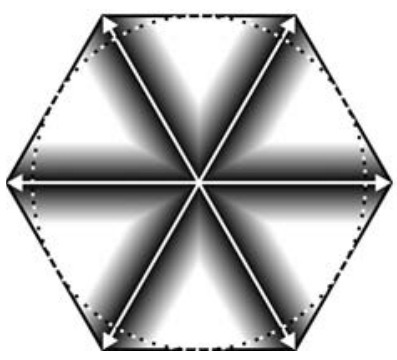

a

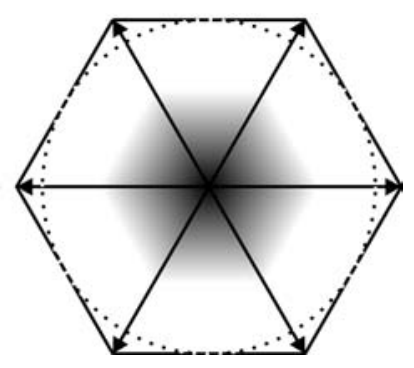

$b$
Fig. $3 S V M$ vector voltage reference associated with available DC-link current acquisition time (darker grey levels correspond to lower available acquisition times) for standard SVM routine $a$ Reference is near or in same direction of one of six fundamental vectors

$b$ Low modulation index

Therefore no phase current can be acquired in the whole SVM cycle. The vector diagram of Fig. $3 b$ illustrates this case.

Case C: Overmodulation. When the SVM operates in overmodulation, the state of the switches remains fixed (in a non-zero state) during several consecutive SVM cycles [9]. This unique inverter state allows only one phase current acquisition at each SVM cycle [8].

Solutions for cases A and B can be found in [1-8]. In most of these works, the phase current reconstructions are accomplished by modifying the original SVM switching pattern. These SVM-PMs consist of changing the switching times in order to allow the required DC-link current acquisitions.

Although many different SVM-PMs have been proposed, a systematic procedure to perform the SVM-PM for the whole modulation range (including overmodulation), based on predefined design rules, is not found in the reviewed literature. In the present paper, a procedure to perform modifications to the original SVM pattern is proposed.
These modifications are designed based on the following rules:

1 The number of switch commutations must be kept unmodified, except in overmodulation mode for which this is not possible. This avoids increasing the commutation power losses.

2 The average phase voltages in each SVM cycle must be kept unmodified (except in overmodulation). In overmodulation mode, only the average phase voltage in two consecutive cycles is kept unmodified. This reduces the voltage and current harmonics.

3 The symmetry of the SVM pulses should be perturbed as low as possible. This reduces the high-frequency harmonics. 4 When a modification is introduced, two phase currents must be acquired in a single SVM cycle during its first half period.

5 The computation complexity needed to perform the modifications must be maintained as low as possible.

The proposed SVM-PM procedure begins with an identification of the operating case (A, B or C). Then, the SVM pattern is modified as follows:

\section{SVM-PM for case A:}

Figure $4 a$ shows an illustration of the SVM-PM technique proposed in the present work for case A. First, the switching pulses are ordered by time duration. Then, the second and third pulses are shifted to the left and right, respectively. Finally, the switching sequences are restored to their original order. With these modifications, a second phase current can be acquired in the first half of the SVM cycle. The average voltage value in each single cycle remains unchanged. Furthermore, the number of switch commutations is not increased and the symmetry of the switching pulses is slightly perturbed (increasing the high-frequency harmonics). Figure $4 b$ shows the SVM-PM as a vector diagram, where the additional states are drawn.

\section{SVM-PM for case $B$ :}

The procedure for case B is similar to that of case A. In this case, the three pulses have almost equal durations; hence,
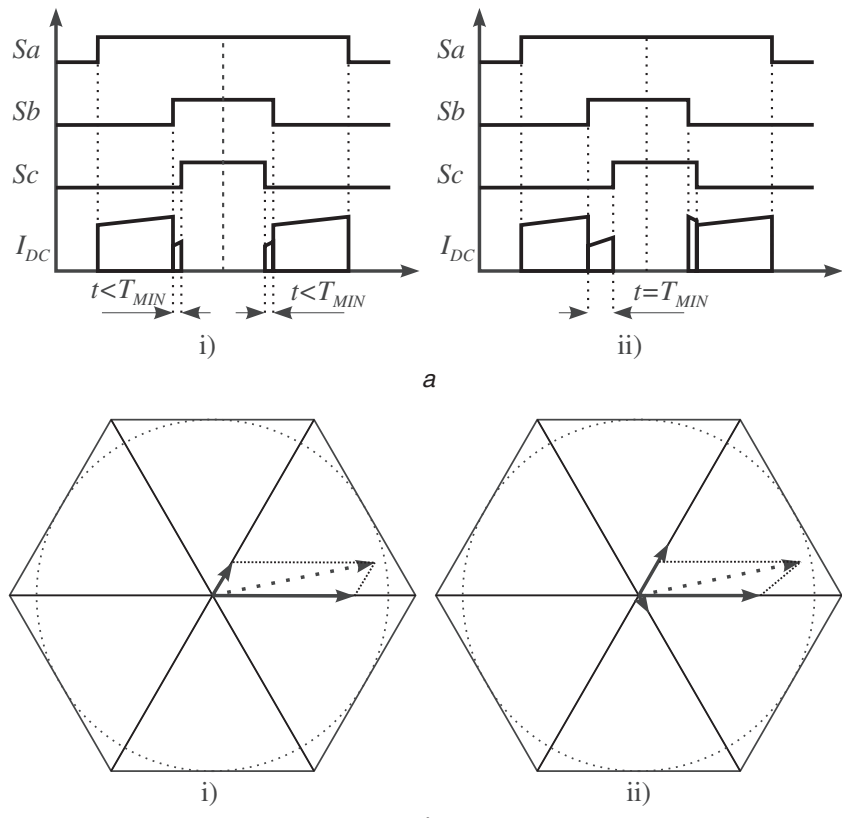

Fig. 4 Proposed SVM-PM for case A

$a$ Switching state sequence example and its corresponding DC-link current

$b$ Corresponding space vectors (dotted line: resultant voltage; solid line: vector components)

Case (i) is original sequence and case ii) is proposed SVM-PM 


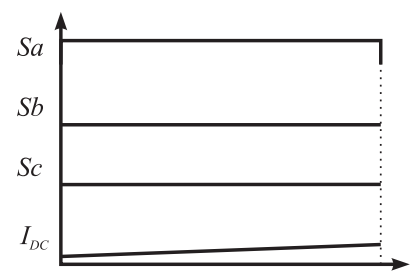

i)

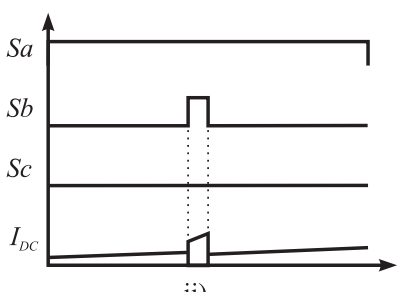

ii)

a
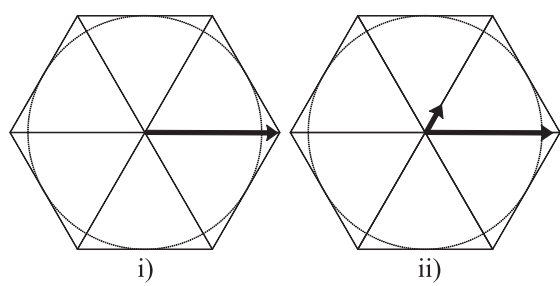

$b$

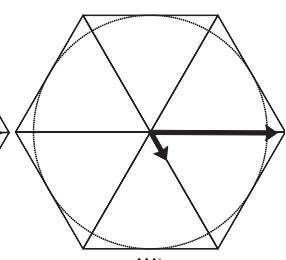

iii)

Fig. 5 Proposed SVM-PM for overmodulation

$a$ Switching sequence example and corresponding DC-link current $b$ Corresponding space vectors

Case (i) is original sequence. Cases (ii) and (iii) are proposed SVMPM, with alternations in consecutive cycles to avoid phase distortion

the first and third switching pulses are shifted to the left and right, respectively.

\section{SVM-PM for case C:}

The solution for this case is more complex, because in overmodulation the inverter remains in a fixed state for several switching cycles. Hence, if only one state is present in the whole SVM cycle, only one phase current can be reconstructed from the DC-link sensor. The only way to allow any other phase current acquisition is by adding an extra active state. This extra state cannot be compensated for in the same way as in the other cases, so the RMS value of the fundamental voltage is reduced. Figure 5 illustrates the proposed SVM-PM for the overmodulation mode. As the added state cannot be compensated for, the two adjacent vectors are alternated to avoid affecting the resultant average phase, as shown in Fig. $5 b$, (diagrams (ii) and (iii)). However, the average voltage magnitude is reduced. In conclusion, in overmodulation it is not possible to modify the SVM pattern without reducing the output RMS voltage value.

\subsection{Current estimation}

Observers are strategies commonly used to estimate the magnetic flux needed by many high-performance IM control strategies [14]. Usually, the observer inputs are the stator voltages and currents, and the rotor speed. Observers can be improved by reducing the number of measured variables, reducing then the number of sensors (e.g. speed sensor). In [12], an adaptive observer that can estimate the rotor flux and the shaft speed of an IM, based on the stator voltages and currents was proposed. The stability of this observer was widely studied and improved in subsequent papers (e.g. $[15,16])$.

In this Subsection, the state observer used to estimate the stator currents and the other motor state variables is described. This observer uses a model of the induction motor in a stationary reference frame. The input variables are the stator voltages. The stator currents and the rotor fluxes are the state variables, while the stator currents are the outputs. Considering that the rotor speed $\left(\omega_{r}\right)$ varies slowly as compared with the electric time constants, the motor model results in a linear time variant system with $\omega_{r}$ as one of its parameters. Hence, the induction motor model is the following [12]:

$$
\begin{aligned}
\frac{d}{d t} \boldsymbol{x}(t) & =\boldsymbol{A}\left(\omega_{r}\right) \boldsymbol{x}(t)+\boldsymbol{B} \boldsymbol{u}(t) \\
\boldsymbol{y}(t) & =\boldsymbol{C} \boldsymbol{x}(t)
\end{aligned}
$$

where

$$
\begin{gathered}
\boldsymbol{A}\left(\omega_{r}\right)=\left[\begin{array}{cc}
-a \boldsymbol{I} & \frac{M}{b T_{r}} \boldsymbol{I} \\
\frac{M}{T_{r}} \boldsymbol{I} & -\frac{1}{T_{r}} \boldsymbol{I}
\end{array}\right]+\omega_{r}\left[\begin{array}{cc}
0 & -\frac{M}{b} \boldsymbol{J} \\
0 & \boldsymbol{J}
\end{array}\right] \\
\boldsymbol{x}=\left[\begin{array}{c}
\boldsymbol{I}_{s} \\
\lambda_{\mathbf{r}}
\end{array}\right], \boldsymbol{B}=\left[\begin{array}{c}
\frac{L_{r}}{b} \boldsymbol{I} \\
0
\end{array}\right], \boldsymbol{C}=\left[\begin{array}{ll}
\boldsymbol{I} & 0
\end{array}\right] \\
a=\frac{L_{r}^{2} R_{s}+M^{2} R_{r}}{b L_{r}}, b=L_{s} L_{r}-M^{2}, \boldsymbol{I}=\left[\begin{array}{ll}
1 & 0 \\
0 & 1
\end{array}\right], \\
\boldsymbol{J}=\left[\begin{array}{cc}
0 & -1 \\
1 & 0
\end{array}\right], T_{r}=\frac{L_{r}}{R_{r}}
\end{gathered}
$$

The motor parameters are the stator and rotor resistances, $R_{s}$ and $R_{r}$, the self inductances of the stator and rotor, $L_{s}$ and $L_{r}$, and the mutual inductance $M$. This model is used in the closed-loop observer with the stator current estimation error as the correcting term [14]. Mathematically,

$$
\frac{d}{d t} \hat{\boldsymbol{x}}(t)=\boldsymbol{A}(t) \hat{\boldsymbol{x}}(t)+\boldsymbol{B} \boldsymbol{u}(t)+\boldsymbol{G}\left(\hat{\boldsymbol{i}}_{s}-\boldsymbol{i}_{s}\right)
$$

where $(\wedge)$ stands for estimated variables, and the gain matrix $\boldsymbol{G}$ is defined in order to assure the observer convergence and to improve its performance [14].

It can be demonstrated, using Lyapunov stability theory, that the rotor speed can be estimated using the following proportional plus integral (PI) adaptation law [12]:

$$
\hat{\omega}_{r}=k_{P}\left(e_{i d s} \hat{\lambda}_{q r}-e_{i q s} \hat{\lambda}_{d r}\right)+k_{I} \int\left(e_{i d s} \hat{\lambda}_{q r}-e_{i q s} \hat{\lambda}_{d r}\right)
$$

where

$$
e_{i d s}=\hat{i}_{d s}-i_{d s}, e_{i q s}=\hat{i}_{q s}-i_{q s}
$$

This full-order observer estimates all the machine state variables. These estimations are available in the controller and can be used to implement a high-performance drive, as will be described in Section 3.

The observer input variables, the phase voltages, can be reconstructed at every SVM cycle using a single DC-link voltage sensor and the inverter switching states. The equations used for the voltage reconstruction are similar to those used to compute the SVM switching times [10], but the unknown variable is the output voltage vector instead of the switching times [13]. The voltage reconstruction technique is well explained in the available literature (e.g. [13]), so further description will be omitted from this paper.

In the proposed scheme, the observer works in closed loop during the controller computation cycles when the phase currents are reconstructed. Conversely, when the current reconstruction cannot be performed as in any of the above mentioned cases (A, B or $\mathrm{C}$ ), the processor does not carry out the SVM-PM at every SVM cycle. The SVM-PM is only performed once every $N$ cycles (where $N$ is a parameter that depends on the implementation). While the SVM-PM is not carried out $(N-1$ cycles), the observer 


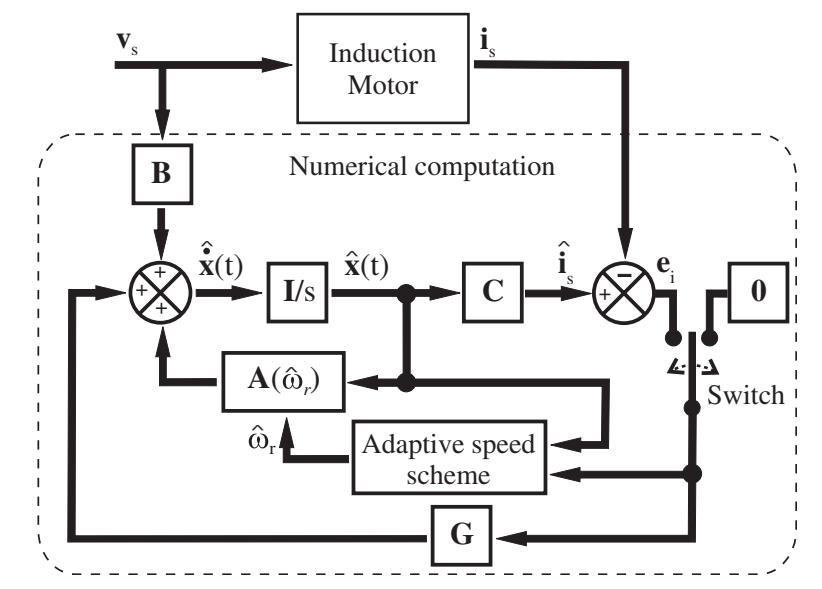

Fig. 6 Adaptive observer block diagram

works in open-loop mode. Figure 6 shows a block diagram of the adaptive observer, where the switch represents the commutation between its closed- and open-loop modes of operation.

If the motor model and its parameters are correct, the state variables in the observer will evolve as the real ones even if it works in open loop for short periods (compared with the electromagnetic time constants). The adaptive speed estimation scheme needs the current error feedback. However, if the open-loop operation time is short enough, then the speed estimation will not be significantly affected. Hence, the observer provides accurate electromagnetic states and speed estimations at every cycle although the currents are reconstructed periodically, once every $N$ cycles.

Exhaustive numerical simulations were performed in order to verify the observer estimation accuracy working in commutated mode (i.e. alternately in closed-loop and openloop mode). The observer feedback was switched by a $1 \mathrm{kHz}$ periodic signal with a programmable duty cycle $(d)$. The duty cycle of the feedback represents the ratio of the number of cycles the observer operates in closed-loop mode (the phase currents are reconstructed) with respect to the number of cycles at which the observer operates in open-loop mode. Mathematically, $d=1 / N$.

The commutation high frequency is filtered out by the integral term of the adaptive scheme (3). The effective feedback gain is affected by the duty cycle of the current measurement. For different values of $k_{I}$ and $d$, a same $d k_{I}$ product produces almost identical simulation results.

\subsection{Analysis of SVM-PM effects}

In this Subsection, a quantitative analysis of the negative effects of the SVM-PM is presented. This SVM-PM must be performed to allow the phase current reconstructions, and it is reduced with the strategy proposed in this paper.

Figure $7 a$ shows a numerical analysis that demonstrates how the RMS value of the fundamental waveform is affected by the SVM-PM, with the inverter working in overmodulation (six-step mode), for different values of $N$ and different minimum acquisition times $\left(T_{M I N}\right)$. In this Figure, $T_{M I N}$ varies from 0 to $20 \%$ of the SVM period ( for this particular implementation, the SVM frequency is $15 \mathrm{kHz}$, hence the $T_{M I N}$ range is $\left.0-13 \mu \mathrm{s}\right)$. The output voltage reduction is considerably high (up to $\sim 10 \%$ ) if the SVM-PM is performed at every cycle $(N=1)$, and can be significantly reduced if the SVM-PM is omitted $(N \rightarrow \infty)$. The maximum voltage increment due to overmodulation is near $10 \%$ [9]. This voltage increment can be lost completely unless the proposed technique is used.
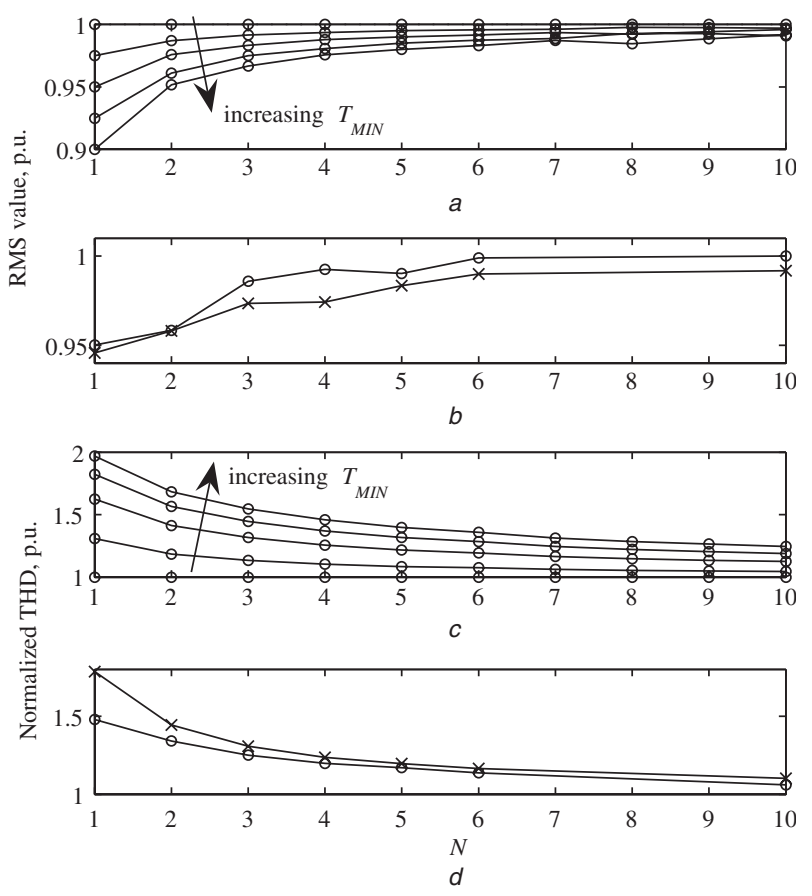

Fig. 7 Numerical computations and experimental validation that represent the negative effects of SVM-PM

$a$ Output RMS voltage during six-step operating mode against $N$ and $T_{M I N}$

$b$ Experimental results for phase currents (o) and voltages (x)

$c$ Normalised THD, with respect to its value without SVM-PM, against $N$ and $T_{M I N} . T_{M I N}$ is varied from 0 to $20 \%$ of SVM period, in steps of $5 \%$

$d$ Experimental results of THD for phase currents (o) and voltages (x)

The SVM-PM also produces an extra high frequency distortion that increases the audible noise and the power losses, because the symmetry of the SVM waveform is affected. Figure $7 c$ shows a numerical computation of the total harmonic distortion (THD) of the output voltage for different values of $N$. The THD is normalised with respect to its value without SVM-PM. The THD was computed by evaluating numerically the Fourier series of the ideal output voltage waveform, from $0 \mathrm{~Hz}$ to $30 \mathrm{kHz}$ (twice the SVM frequency).

The SVM-PM effects predicted by the numerical computations were validated experimentally with the implemented drive (described in the next section). Figures $7 b$ and $d$ show the output RMS value and the THD of the phase currents and voltages as a function of $N$. These results demonstrate the advantages of the proposed scheme, reducing significantly the SVM-PM negative effects. The value of $T_{M I N}$ used in the experimental tests is $7 \mu$ s.

\section{Prototype implementation and experimental results}

\subsection{Description of the prototype}

An IM drive based on a DSP (TMS320F2812) was implemented to validate the proposed strategies. The used IM parameters are given in Table 2. The DSP runs a standard rotor-flux oriented control (FOC) algorithm, based only on DC-link measurements. Figure 8 shows a block diagram of the proposed scheme. The DSP performs the DC-link current and voltage acquisitions, the computation of the observer, the FOC algorithm and the SVM-PM.

The power stage was implemented with a MOSFET inverter that feeds a low-voltage induction motor. The 
Table 2: Induction machine parameters

\begin{tabular}{ll}
\hline Parameter & Value \\
\hline$P(\mathrm{~kW})$ & 1.25 \\
$V(\mathrm{~V} \mathrm{rms})$ & 25 \\
$F(\mathrm{~Hz})$ & 83 \\
Rated speed $(\mathrm{rpm})$ & 2500 \\
$R_{r}(\mathrm{~m} \Omega)$ & 34.7 \\
$R_{s}(\mathrm{~m} \Omega)$ & 69.7 \\
$M(\mathrm{mH})$ & 2.66 \\
$L_{l r}(\mathrm{mH})$ & 0.117 \\
$L_{l s}(\mathrm{mH})$ & 0.117 \\
\hline
\end{tabular}

DC-link current is measured with an SR $(5 \mathrm{~m} \Omega)$ and the DC-link voltage is measured using a resistive network. Both measurements are acquired by the DSP on-chip ADC.

The reconstruction of the phase currents is performed using the DC-link current acquisitions and the states of the inverter switches from the SVM module. The phase voltages are also computed using the inverter states and DC-link voltage acquisitions.

The SVM module is implemented using a method that allows a linear operation of the inverter, even in overmodulation mode. Overmodulation mode allows a higher fundamental output voltage value with the same voltage source. The implemented overmodulation technique is similar to the one proposed in [9].

The phase current and voltage values feed the adaptive observer that estimates the IM state variables. The observer current estimations are used as feedback variables of the current controllers, the estimated rotor vector flux components $\left(\lambda_{q}\right.$ and $\left.\lambda_{d}\right)$ are used to compute the flux position $\theta_{\lambda}$ required by the FOC algorithm, and the estimated speed is used to feed back to the speed-control loop. In this implementation, the maximum open-loop observer cycles is limited to three (hence, $N=4$ ).

The current and speed control loops use PI (proportional-integral) controllers with an anti-wind-up scheme based on conditional integration [17] for avoiding integrator wind-up.

The adaptive observer estimates the rotor speed based on the motor EMF. At low speeds, this EMF is very low as compared with the voltage drop of the stator resistance. Hence, the speed estimation is too inaccurate because of the model uncertainty and measurement errors. The same occurs with other EMF-based speed estimation schemes [11]. In the present implementation, the lower speed limit is
0.05 p.u. Hence, from zero to \pm 0.05 p.u. a scalar $\mathrm{V} / \mathrm{f}$ control algorithm is used, and above that speed the FOC algorithm starts running. A weighted sum of the voltage references is used to smooth the transition between the two control strategies.

The observer differential equations are solved in real time using a second-order Runge-Kutta (R-K) method [18]. This method produces considerably better results than Euler's method with the time step used $(6.66 \mu \mathrm{s})$. A fourth order R$\mathrm{K}$ method was also evaluated but it produced similar results as the second-order $\mathrm{R}-\mathrm{K}$ but with more computation demand.

\subsection{Experimental results}

Figures 9-13 show some relevant experimental results obtained with the implemented IM FOC drive using the proposed strategy. The drive operates in closed speed loop based only on DC-link measurements.

Figure 9 shows, a typical DC-link current sensor signal during a commutation. The transient oscillations are produced by the high slew-rate of the MOSFET current commutations, the parasitic inductances of the SR and the DC-link layout. Although these transient oscillations are high, they extinguish very rapidly (less than $6 \mu \mathrm{s}$ ), so if the current acquisitions are performed after a certain time $\left(T_{M I N}\right)$, the acquisition errors can be negligible. The transient oscillations can be filtered out by using lowpass filters, as shown in Fig. 9. The calibration of the acquisition system is not much more complex than the calibration of a standard phase current sensor. Setting a higher $T_{M I N}$ will not affect significantly the performance of the drive, so it could be over-dimensioned. Other types of current sensors may produce lower transient oscillations levels.

Figure 10 shows the phase current waveforms for different modulation indexes. Figure $10 a$ shows the current for the case of very low modulation index $(=0.05)$, Fig. $10 b$

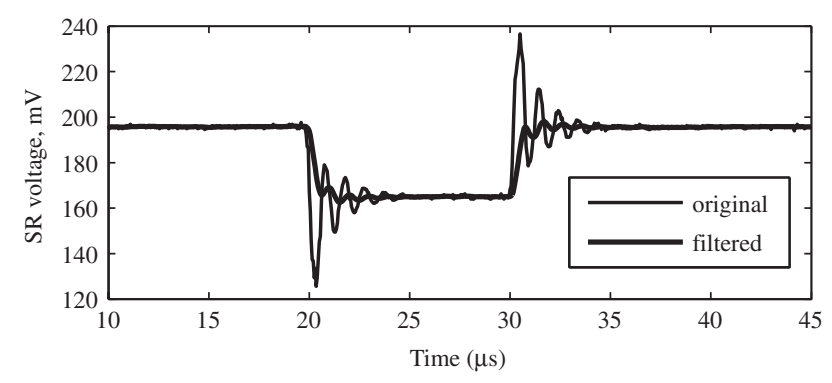

Fig. 9 DC-link current sensor signal during two commutations Thin line is original sensor signal and thick line is same signal filtered by first-order filter (RC) with cutoff frequency of $400 \mathrm{kHz}$

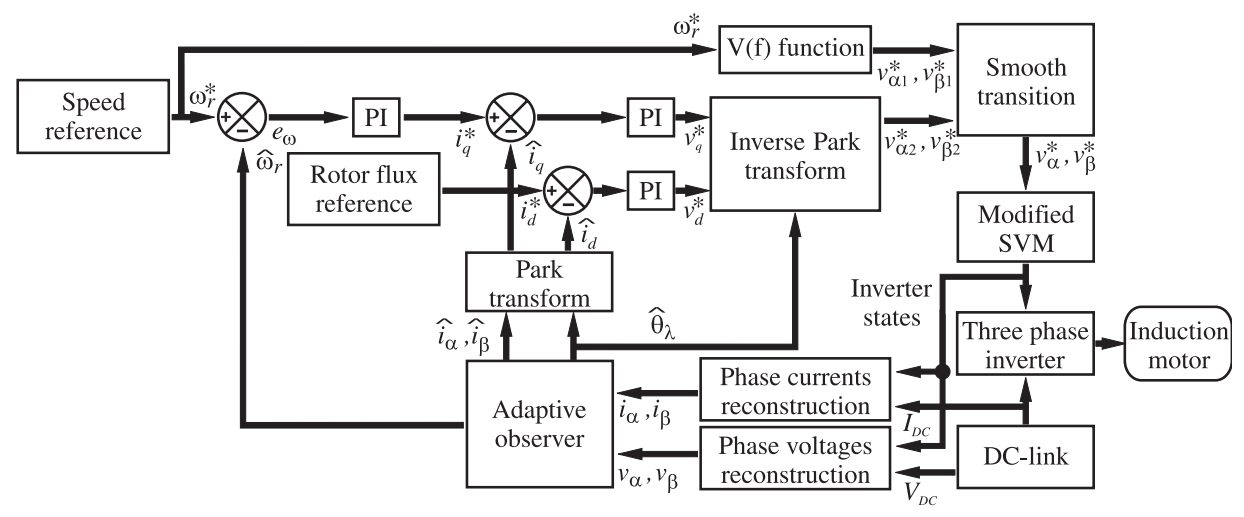

Fig. 8 Block diagram of proposed mechanical-sensorless FOC drive 

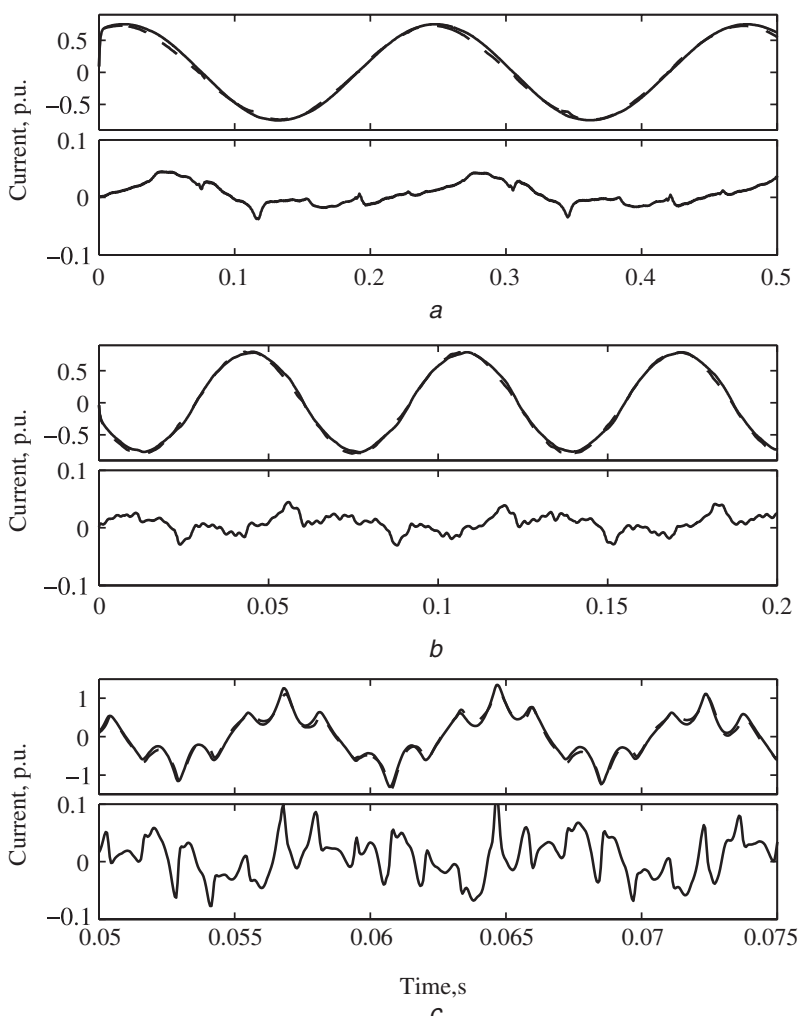

Fig. 10 Experimental results showing estimated (solid line), measured (dashed line) and estimation error (separated subfigures) of current waveform for different modulation indexes

$a$ Very low modulation index $(=0.05)$

$b$ Medium modulation index $(=0.5)$

$c$ Very high modulation index $(=1.0)$
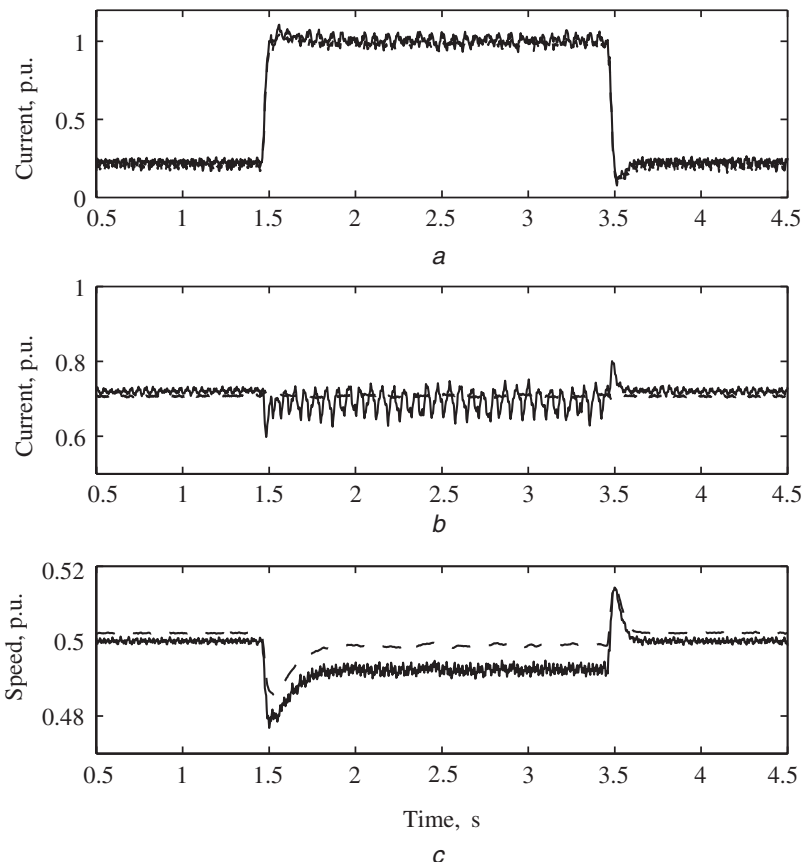

Fig. 11 FOC drive experimental results showing dynamic response for a 100\% load step applied at $1.5 \mathrm{~s}$ and removed at $3.5 \mathrm{~s}$

a $i_{q}$ current

$b i_{d}$ current

$c$ rotor speed

Estimated values are represented by dashed lines and measured values with solid lines
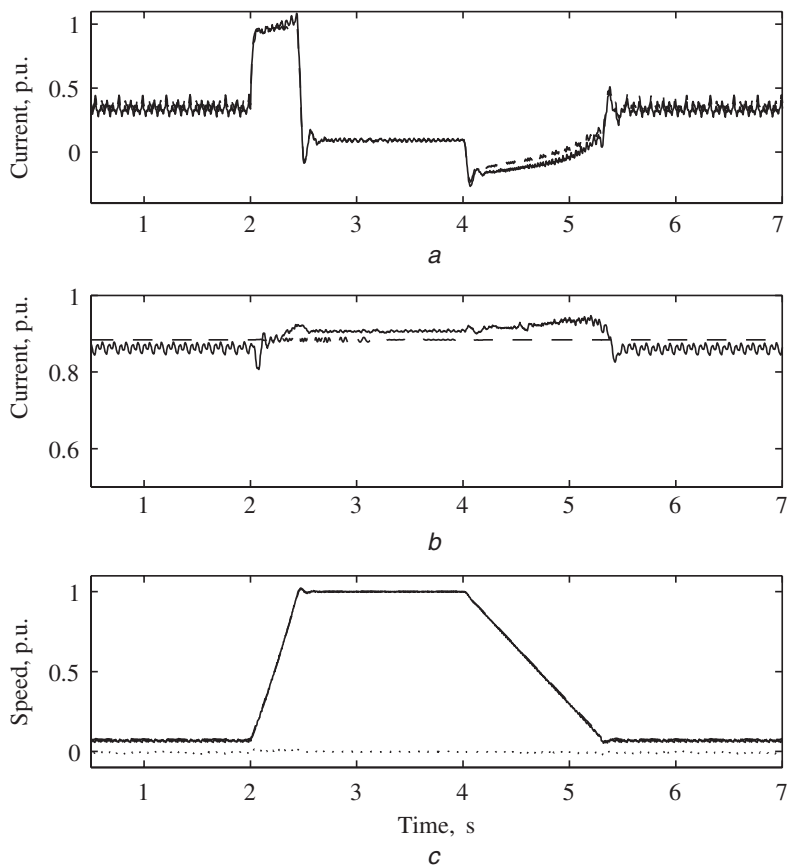

Fig. 12 FOC drive experimental results showing dynamic response of drive, without external load, for speed reference step applied at $2 \mathrm{~s}$ a $i_{q}$ current

$b i_{d}$ current

$c$ rotor speed

Estimated values are represented by dashed lines and measured values by solid lines. Speed error is drawn with dotted line

for medium modulation index $(=0.5)$, and Fig. $10 c$ for very high modulation index (overmodulation). The modulation index is defined in [9]. Phase currents were measured using Hall-effect sensors only for comparison purposes. The measured current, estimated current and the estimation error are shown as subplots. From these plots, it can be supposed that the current estimations are accurate enough to feed back to the FOC prototype. In the following tests, this supposition is confirmed.

Dynamic response tests are presented to demonstrate the behaviour of the current estimation scheme and the performance of the FOC drive. A commercial drive with a $5 \mathrm{~kW}$ induction machine was used to apply external load.

Figure 11 shows the dynamic response for the application of a step of external load torque. A 100\% rated load is applied at $1.5 \mathrm{~s}$ and removed at $3.5 \mathrm{~s}$. The Figure shows the fast response of the drive, necessary to counteract the external load, keeping the motor speed very close to its reference ( 0.5 p.u.). Figures $11 a$ and $b$ show the good dynamic and static response of the $i_{q}$ and $i_{d}$ current estimations. The current ripple is caused by the non-ideal voltage waveforms of the inverter (caused by the deadtimes, commutation times and voltage drops). The estimated rotor speed is compared with the speed measured with an optical incremental encoder. The speed estimation error at rated load is less than $2 \%$.

Figure 12 shows the dynamic response of the drive for a speed reference step without external load. The motor is accelerated from very low speed $(0.05$ p.u. $)$ to rated speed (1 p.u.) and back to very low speed ( 0.05 p.u.). The dynamic response shown by the drive is comparable to standard FOC drives. The $i_{q}$ and $i_{d}$ current estimations show a small error in the transients, which is reduced in the steady state. The estimated speed error is also very small, even during the acceleration. The braking torque is lower than the motoring torque only because of braking unit limitations. 

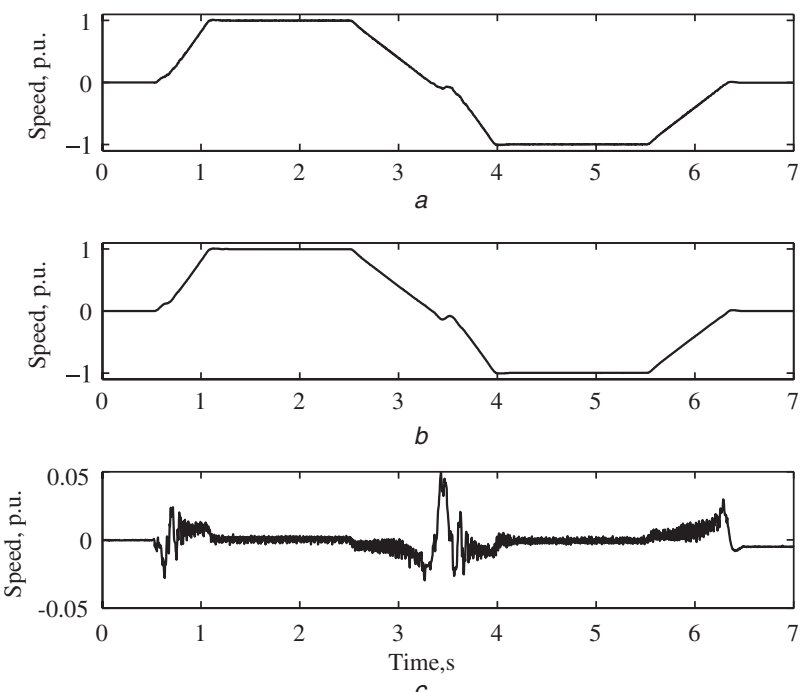

Fig. 13 FOC drive experimental results showing dynamic response for forward-reverse speed operation without external load $a$ Estimated speed

$b$ measured speed

$c$ estimation error

Figure 13 shows the dynamic response of the speed estimation during a forward-reverse speed operation. The plots show a very small (less than $1 \%$ ) speed error at steady state, which is higher during the accelerations, as expected from the considerations used in the design of the speed estimation scheme [12]. The estimation error is higher when the speed is close to zero, as was mentioned above, so in this speed region the drive is controlled by a scalar V/f strategy. The presented experimental results demonstrate a high performance comparable to sensorless drives that measure directly the phase currents.

The robustness of the implemented drive can be improved with the addition of a parameter estimation scheme. This scheme can be based on the same electrical variables (voltages and currents), as is proposed in [12]. The analysis and implementation of these kinds of strategies is beyond the scope of this paper. The authors consider that it would be a valuable improvement for implementing very low-cost, high-performance commercial drives, using the scheme proposed in this paper.

\section{Conclusions}

A complete solution for an induction motor (IM) mechanical-sensorless field-oriented controller (FOC) drive has been presented. This drive uses only single current and single voltage DC-link sensors to operate the FOC in closed speed-loop ( from 0.05 p.u. speed).

The main contribution of this work is the improvement of the single current sensor technique to compute the IM phase currents. The proposed scheme uses simultaneously a reconstruction technique based on the DC-link current acquisition and a full-order state observer. A procedure to perform the space vector modulation pattern modification (SVM-PM), needed to reconstruct the phase currents from the DC-link sensor is proposed, valid for the whole modulation range. The negative effects of this SVM-PM are reduced by using the observer currents estimations.

It has been demonstrated quantitatively, using numerical computations and experimental tests, that the observer allows significant reduction of the negative influence of the SVM-PM in the drive performance, particularly the RMS output-voltage reduction and the introduction of highfrequency harmonics.

The presented experimental results show the accuracy and good dynamic performance of the current and speed estimations, even in fast speed transients and high external loads. The experimental results demonstrate that the implemented drive has a high performance, similar to that of a conventional FOC drive, which uses individual phase current sensors.

The sensor count reduction achieved represents a significant cost reduction in the implementation of lowpower high-performance induction motor drives.

\section{Acknowledgements}

The authors thank the Texas Instruments DSP University Program staff for the donation of DSP development boards. This allowed implementing experimental prototypes, to include experimental results in this paper and also to approach our R\&D team to a possible transference to industry. This work was supported by Universidad Nacional de Río Cuarto (UNRC), Universidad Nacional del Sur (UNS), ANPCyT and CONICET.

\section{References}

1 Texas Instruments, Three phase current measurements using a single line resistor on the TMS320F240, Lit. Num. BPRA077, 1998

2 Green, T.C., and Williams, B.W.: 'Derivation of motor line-current waveforms from DC-link current of an inverter', IEE Proc., Electr. Power Appl., 1989, 136, pp. 197-204

3 Xue, Y., Xu, X., Habetler, T.G., and Divan, D.M.: 'A stator fluxoriented voltage source variable-speed drive based on DC link measurement', IEEE Trans. Ind. Appl., 1991, 27, pp. 962-969

4 Blaabjerg, F., and Pedersen, J.K.: 'An ideal PWM-VSI inverter using only one current sensor in the DC-link'. Proc., 1994, (IEEE PEVD), pp. $458-464$

5 Lee, W., Lee, T., and Hyun, D.: 'Comparison of single-sensor current control in the DC link for three-phase voltage-source PWM converters', IEEE Trans. Ind. Electron., 2001, 48, (3), pp. 491-505

6 Wolbank, T.M., and Macheiner, P.: 'An improved observer-based current controller for inverter fed AC machines with single DC-link current measurement'. Proc. IEEE PESC, 2002, pp. 1003-1008

7 Joo, H.G., Kim, C., Shin, H., and Youn, M.: 'Detection of threephase currents in space-vector PWM inverters with only one DC link current sensor'. Proc. IEEE IECON, 1996, pp. 127-132

8 Blaabjerg, F., and Pedersen, J.K.: 'A new low-cost, fully faultprotected PWM-VSI inverter with true phase-current information', IEEE Trans. Power Electron., 1997, 12, pp. 187-197

9 Lee, D.Ch., and Lee, G.-M.: 'A novel overmodulation technique for space-vector PWM inverters', IEEE Trans. Power Electron., 1998, 13, pp. 1144-1151

10 Holtz, J.: 'Pulsewidth modulation - a survey', IEEE Trans. Ind Electron., 1992, 39, (5), pp. 410-420

11 Rajashekara, K., Kawamura, A., and Matsuse, K.: 'Sensorless Control of AC Motor Drives' (IEEE Press, 1996)

12 Kubota, H., Matsuse, K., and Nakano, T.: 'DSP-based speed adaptive flux observer of induction motor', IEEE Trans. Ind. Appl., 1993, 29, pp. $344-348$

13 Texas Instruments, Digital Motor Control Software Library, Lit. Num. SPRA485A, 2003

14 Verghese, G.C., and Sanders, S.T.: 'Observers for flux estimation in induction machines', IEEE Trans. Ind. Electron., 1988, 35, (1), pp. 85-94

15 Suwankawin, S., and Sangwoungwanich, S.: 'A speed-sensorless IM drive with decoupling control and stability analysis of speed estimation', IEEE Trans. Ind. Electron., 2002, 49, (2), pp. 444455

16 Hinkkanen, M., and Luomi, J.: 'Stabilization of regenerating-mode operation in sensorless induction motor drives by full-order flux observer design', IEEE Trans. Ind. Electron., 2004, 51, (6), pp. $1318-1328$

17 Visioli, A.: 'Modified anti-windup scheme for PID controllers', IEE Proc. Control Theory Appl., 2003, 150, (1), pp. 49-54

18 Press, W.H., Teukolsky, S.A., Vetterling, W.T., and Flannery, B.P.: 'Numerical Recipes in C' (Cambridge University Press, USA, 1994) 